\title{
RESULTS OF THE MONITORTNG OF A VERY STRONG WATER MASER EVENT IN W49N
}

\author{
E. SCALISE JR., G.M. PACHECO, A.M. GOMEZ BALBOA 1 AND Z. ABRAHAM 2 \\ SCT - INPE, CP 515, 12201 S.J.Campos, São Paulo; \\ ${ }^{1} \mathrm{CNPq}-\mathrm{ON}, \mathrm{CP} 23002,20921$ Rio de Janeiro; \\ 2USP - IAG, CP 9638, 04301 São Paulo. BRASIL
}

\begin{abstract}
Since the discovery of the $6_{16}-5_{23}$ rotational transition of interstellar water in maser emission, several hundred sources have been discovered. Their profiles are very variable, presenting correlated variability (Gammon, 1976) or anticorrelated varlability (Cesaroni, 1990). Several models have been proposed to explain this variability. Strong Galactic masers, such as W49 and W51 (Kylafis et a1. 1991), and also an extremely strong outburst detected by Abraham et al. (1981) in Orion, could not be fully explained based on current models. We have monitored from October 1989 to July 1991 a very strong water vapour eruption in the $+28 \mathrm{~km} \mathrm{~s}^{-1}$ feature, originated from W49N. Here we present the results and discuss the possible correlated variability of this feature with the one that appeared at $+62 \mathrm{~km} \mathrm{~s}^{-1}$.
\end{abstract}

\section{Equipment and observations}

The observations were carried out with the Itapetinga $13.7 \mathrm{~m}$ radiotelescope. At $22 \mathrm{GHz}$ the HPBW is 4.2 arc min. The system temperature is of about $1000 \mathrm{~K}$. The signal is detected by an acoustooptical spectrometer with 1000 channels of $40 \mathrm{kHz}$ each.

The spectra were initially corrected to account for the change of atmosphere attenuation. In order to eliminate any gain fluctuation the spectra were normalized, one against the other, using the average antenna temperature of an "undisturbed" region situated in the velocity range from -20 to $-30 \mathrm{~km} \mathrm{~s} \mathrm{~s}^{-1}$. We have calculated the uncertainties in the antenna temperature to be of the order of $10 \%$.

\section{Discussion and results}

In Figure 1 we can see that the $+28 \mathrm{~km} \mathrm{~s}^{-1}$ feature was observed for the first time, above the minimum detectable temperature, on October 1989, reaching a relative maximum by April 1990. Superimposed to this activity a very strong event happened in the region causing the emission to rise, almost linearly, three folds in 50 days. This level of emission remained high during the next 200 days, presenting some fluctuations. Polarization measurements carried out at this phase showed no percentage of linear polarization larger than $10 \%$. 
The decay phase, to the pre-flare level, was slower than the rise time and lasted for 90 days. The return to the zero level is hapenning in a similar way as of its rise but it has not yet been achieved.

Assuming the radiation to be isotropic, the line width to be of the order of our spectrometer resolution, and the source to be placed at $14 \mathrm{kpc}$, at its maxima, this event reached the intensity of 0.073 solar luminosities, becoming one of the strongest water vapour eruptions ever recorded in the Galaxy, but still 4 orders of magnitude weaker than extragalactic megamasers.

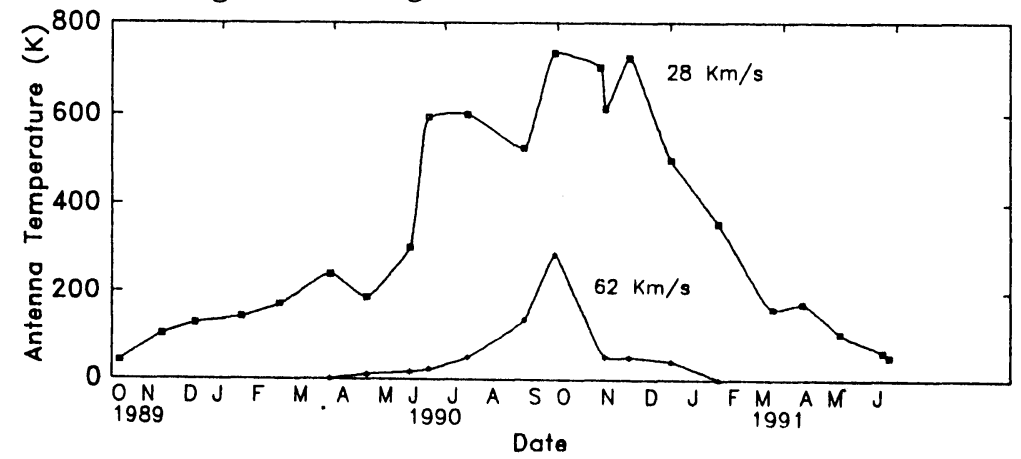

Figure 1 - Time evolution of the $+28 \mathrm{~km} \mathrm{~s}^{-1}$ and $+62 \mathrm{~km} \mathrm{~s}^{-1}$ features of W49N covering the period from October 1989 to July 1991.

The other feature at $+62 \mathrm{~km} \mathrm{~s}^{-1}$ rised slowly during 145 days, decreased in only 30 days to a quiescent level and disappeared completely after 80 days. The overall shape of this event disagrees with the diffusive model of Burke et al. (1978).

From the analysis of the VLBI map of this region (Walker et al 1982), it looks possible that these two features correspond to two spots lying 0.230 milliarcsecond apart. If the excitation source of both features is spatially coincident with the $+28 \mathrm{~km} \mathrm{~s}^{-1}$, its front of excitation has to propagate at a velocity larger than $2000 \mathrm{~km} \mathrm{~s}^{-1}$ to reach the $+62 \mathrm{~km} \mathrm{~s}^{-1}$ spot, what will cause the ionization of all surrounding molecules. But if it lies somewhere between the spots, the velocity of the front would be such to interact successively with the two features without destroying the molecules of the medium.

Acknowledgement: The authors wish to express their gratitude to the Itapetinga Radio Observatory staff. This work was partially supported by the Brazilian agencies FAPESP, CAPES and CNPq.

References:

Abraham, Z•, Cohen, N.L., Opher, R•, Raffaelli, J.C. \& Zisk, S.H.

(1981) Astron. Astrophys., 100, L10.

Burke, B.F., Giufrida, T.S.\& Haschick, A.D. (1978), Ap.J., 226, L21.

Cesaroni, R. (1990), Astron. Astrophys., 233, 513.

Gammon, R.H. (1976), Astron. Astrophys., 50, 71.

Kylafis, N.D. \& Norman, C.A. (1991), Ap. J., in press.

Walker, R.C., Matsakis, D.N॰ \& Garcia-Barreto, J.A. (1982), Ap.J., $255,128$. 